\title{
A percepção das pacientes submetidas ao tratamento de braquiterapia ginecológica
}

\author{
The perception of patients undergoing gynecological brachytherapy treatment \\ La percepción de las pacientes sometidas a tratamiento de braquiterapia ginecológica
}

Recebido: 11/11/2021 | Revisado: 16/11/2021 | Aceito: 16/11/2021 | Publicado: 19/11/2021

\author{
Ianna Mara Borges de Sousa \\ ORCID: https://orcid.org/0000-0003-2821-0811 \\ Universidade Ceuma, Brasil \\ E-mail: borgesianna@hotmail.com \\ Marluce Sampaio Nobre Barbosa \\ ORCID: https://orcid.org/0000-0001-7276-6521 \\ Universidade Ceuma, Brasil \\ E-mail: marluce.nobre@ceuma.br \\ Haigle Reckziegel de Sousa \\ ORCID: https://orcid.org/0000-0002-5803-2289 \\ Universidade Ceuma, Brasil \\ E-mail: haiglereck3@gmail.com \\ Felype Hanns Alves de Medeiros \\ ORCID: https://orcid.org/0000-0001-5026-1995 \\ Universidade Estadual do Pará, Brasil \\ E-mail: felypehanns@hotmail.com \\ Érika Ferreira Tourinho \\ ORCID: https://orcid.org/0000-0003-2167-930X \\ Universidade Ceuma, Brasil \\ E-mail: tourinhoerika@gmail.com \\ Patrícia dos Santos Silva Queiroz \\ ORCID: https://orcid.org/0000-0002-9587-1786 \\ Universidade Ceuma, Brasil \\ E-mail: patriciasqueiroz@gmail.com
}

\begin{abstract}
Resumo
Introdução: A braquiterapia é um tipo de radioterapia em que a fonte radioativa é colocada dentro da área a ser tratada ou junto a ela. A inserção pode ser temporária ou permanente. Uma das características mais importantes da braquiterapia é que a radiação gerada pela fonte afeta apenas áreas muito próximas do local que será tratado, protegendo assim os tecidos sadios. Objetivo: Conhecer o significado da braquiterapia e a percepção dolorosa de mulheres com câncer ginecológico submetidas à essa terapêutica numa Unidade de Referência em uma cidade do interior do Maranhão. Metodologia: Trata-se de uma pesquisa descritiva exploratória, com abordagem quantitativa. Resultados: Durante os procedimentos de preparo para a braquiterapia, no decorrer e depois da retirada do encerramento do procedimento percebeu-se que os achados em relação a percepção de dor permaneceram os mesmos como ardência, cólica queimação e dor constante. Mesmo com seus benefícios significativos, esse tratamento também apresenta efeitos adversos que podem chegar a ser irreversíveis caso não seja manejado de forma adequada, assim torna-se de extrema importância compreender as fragilidades e os medos apresentados pelas pacientes no que concerne à doença e ao tratamento, auxiliando na adaptação a essa nova condição e sanar as dúvidas, objetivando uma assistência mais eficiente e adequada. Conclusões: Por fim, conclui-se que é necessária uma ampliação da investigação do perfil sociodemográfico e clínico por meio de estudo quantitativo, somente assim será possível estabelecer um panorama mais amplo sobre as mulheres atendidas numa Unidade de Referência.
\end{abstract}

Palavras-chave: Braquiterapia; Enfermagem; Terapêutica.

\begin{abstract}
Introduction: Brachytherapy is a type of radiotherapy in which the radioactive source is placed within or near the area to be treated. The insertion may be temporary or permanent. One of the most important characteristics of brachytherapy is that the radiation generated by the source affects only areas very close to the site that will be treated, thus protecting healthy tissues. Objective: To know the meaning of brachytherapy and the painful perception of women with gynecological cancer submitted to this therapy in a Reference Unit in a city in the interior of Maranhão. Methodology: This is an exploratory descriptive research, with a quantitative approach. Results: During the preparation procedures for brachytherapy, during and after the withdrawal of the closure of the procedure, it was noticed that the findings in relation to pain perception remained the same as burning, burning colic and constant pain. Even with its significant benefits, this treatment also presents adverse effects that may be irreversible if it is not properly maintained, so it is extremely important to understand the weaknesses and fears presented by patients regarding the disease and treatment, helping to adapt to this new condition and answer doubts, more efficient and
\end{abstract}


appropriate assistance. Conclusions: Finally, it is concluded that it is necessary to expand the investigation of the sociodemographic and clinical profile through a quantitative study, only in this way will it be possible to establish a broader overview of the women assisted in a Reference Unit.

Keywords: Brachytherapy; Nursing; Therapeutics.

\section{Resumen}

Introducción: La braquiterapia es un tipo de radioterapia en la que la fuente radiactiva se coloca dentro o cerca del área a tratar. La inserción puede ser temporal o permanente. Una de las características más importantes de la braquiterapia es que la radiación generada por la fuente afecta solo a áreas muy cercanas al sitio que será tratado, protegiendo así los tejidos sanos. Objetivo: Conocer el significado de la braquiterapia y la percepción dolorosa de las mujeres con cáncer ginecológico sometidas a esta terapia en una Unidad de Referencia en una ciudad del interior de Maranhão. Metodología: Se trata de una investigación descriptiva exploratoria, con enfoque cuantitativo. Resultados: Durante los procedimientos de preparación para la braquiterapia, durante y después de la retirada del cierre del procedimiento, se observó que los hallazgos en relación con la percepción del dolor permanecieron iguales que el ardor, el cólico ardiente y el dolor constante. Aun con sus importantes beneficios, este tratamiento también presenta efectos adversos que pueden ser irreversibles si no se mantiene adecuadamente, por lo que es sumamente importante comprender las debilidades y temores que presentan los pacientes respecto a la enfermedad y el tratamiento, ayudando a adaptarse a esta nueva condición y responder dudas, asistencia más eficiente y adecuada. Conclusiones: Finalmente, se concluye que es necesario ampliar la investigación del perfil sociodemográfico y clínico a través de un estudio cuantitativo, solo así será posible establecer una visión más amplia de las mujeres asistidas en una Unidad de Referencia.

Palabras clave: Braquiterapia; Enfermería; Terapéutica.

\section{Introdução}

O câncer segue sendo compreendido como um importante problema de saúde pública. Com base na estimativa realizada pelo Instituto Nacional de Câncer José Alencar Gomes da Silva, para o biênio 2018-2019 houve cerca de 600 mil casos novos de câncer no Brasil. O câncer ginecológico também compreende uma das neoplasias de ovário, endométrio, colo do útero e vulva. O câncer de colo do útero ainda é o quarto mais incidente e a quarta causa de morte por câncer em mulheres (Ramalho et al., 2020) (Silva, 2015).

Os cânceres ginecológicos são chamados de estadiados de acordo com as diretrizes estabelecidas pela Federação Internacional de Ginecologia e Obstetrícia (FIGO), e o seu tratamento depende muito do tamanho, tipo de tumor, estado geral da cliente e localização do tumor. O câncer pode surgir em qualquer parte do corpo, não existe um padrão para isso. Entretanto, alguns órgãos podem ser mais afetados do que outros; e cada órgão, por sua vez, pode ser acometido por tipos diferenciados de tumor, podendo ser mais ou menos agressivos. Os vários tipos de câncer são classificados de acordo com a localização primária do tumor. Exemplo: colo do útero, mama, pulmão (Araujo et al., 2018).

A Neoplasia, também denominada tumor, é uma forma de proliferação celular não controlada pelo organismo, com tendência para a autonomia e perpetuação. A palavra "neoplasia" vem do grego, onde neo = "novo" e plasis = "crescimento, multiplicação celular”. Além disso, as neoplasias podem ser benignas ou malignas, isso depende bastante do potencial e dos danos que exerce ao indivíduo. O câncer pode atingir pessoas de qualquer faixa etária, porém, é mais comum em pessoas adultas, o certo é que, se a doença for diagnosticada cedo é tratada, a sobrevida de um indivíduo canceroso poderá ser bem mais longa, e em certos casos poderá até levar à cura (Araujo et al., 2018).

A braquiterapia é um tipo de radioterapia em que a fonte radioativa é colocada dentro da área a ser tratada ou junto a ela. A inserção pode ser temporária ou permanente. Uma das características mais importantes da braquiterapia é que a radiação gerada pela fonte afeta apenas áreas muito próximas do local que será tratado, protegendo assim tecidos sadios. É utilizada principalmente em tumores ginecológicos como reforço ao tratamento de radioterapia externo já realizado ou como tratamento exclusivo pós-operatório. Pode ser indicada também para tumores de próstata, pele, mama, cabeça e pescoço, entre outros (Araujo et al., 2017).

No entanto, pelo fato de ser o profissional de saúde que está mais perto do paciente, o enfermeiro acaba 
desenvolvendo, além de suas habilidades de assistência, o papel de educador diante do paciente, da sua família e comunidade. Por meio de uma comunicação efetiva, se torna cada vez mais claro se compreender as fragilidades e os medos que os pacientes acabam mostrando diante, tanto da doença quanto do tratamento, e o enfermeiro pode ser esse suporta para auxiliar na adaptação a essa nova condição e esclarecer todas as dúvidas, com vista a oferecer uma assistência mais eficiente e adequada (Silva et al., 2021).

É relevante destacar que um dos profissionais que participam do procedimento de braquiterapia é o enfermeiro, que fica responsável por cuidar da cliente e gerencia o funcionamento da sala, assim como a infraestrutura para que o tratamento ocorra de fato ${ }^{(4)}$.

Portanto, diante do exposto é justificavel explorar a temática citada anteriormente, correspondente a discutir o papel realizado pela enfermagem aos pacientes submetidos ao tratamento de braquiterapia ginecológica, e consequentemente verificar de maneira prática como as pacientes responderam ou respondem sobre esse tratamento, ou seja, os sentimentos, os efeitos colaterais e etc. Assim pergunta norteadora é qual percepção dolorosa de mulheres com câncer ginecológicas submetidas à essa terapêutica numa Unidade de Referência em uma cidade do Interior do Maranhão?

O objetivo geral é conhecer o significado da braquiterapia e a percepção dolorosa de mulheres com câncer ginecológicas submetidas à essa terapêutica numa Unidade de Referência em uma cidade do Interior do Maranhão.

\section{Metodologia}

A presente pesquisa foi feita aos preceitos e respeitando as Normas de Pesquisa Envolvendo Seres Humanos, Resolução 466/12 do Conselho Nacional de Saúde, ocorrendo a pesquisa somente após a aprovação do CEP/UNICEUMA, parecer $n^{\circ} 4.840 .007$.

Trata-se de uma pesquisa descritiva, do tipo exploratória, com abordagem quantitativa, adequando-se ao modelo Standards for Quality Improvement Reporting Excellence 2.0 (SQUIRE), avaliando as condições do procedimento e com intuito de conhecer as intervenções da equipe A pesquisa exploratória cria-se certa aproximação com o objeto de investigação é envolvido levantamento de bibliográfico, entrevistas com componentes diretos do assunto, assim bem como, a análise e compreensão do problema abordado. O método quantitativo busca um estudo mais profundo, crítico e analítico, distinto das pesquisas com bases numéricas (Quintão et al., 2019).

Assim, houve a necessidade de elaboração de formulário para a obtenção dos dados junto às mulheres com câncer ginecológico submetido a esta terapêutica numa Unidade de Referência, que contém equipe de médico oncologista, cirurgiões, enfermeiros, técnicos de enfermagem, composto por área de laboratório e de tratamento radioterápico.

As participantes estão sendo atendidas desde fevereiro do ano de 2021, com diagnóstico de canceres ginecológicos.

Com relação aos critérios de inclusão para presente pesquisas foram considerados pacientes do sexo feminino, submetidas ao tratamento de braquiterapia ginecológica, com idade de 30 a 70 anos, com diagnóstico de canceres ginecológicos e que concordaram em assinar o Termo de Consentimento Livre e Esclarecido (TCLE).

Os critérios de exclusão compuseram de pacientes do sexo masculino, mulheres com idade menor de 30 anos e acima dos 70 anos e com outro diagnóstico de canceres que não sejam ginecológicos e que se negarem a assinar Termo de Consentimento Livre e Esclarecido (TCLE).

A amostra do estudo não foi intencional, sendo obtidos por identificação das pacientes que foram elegíveis por meio dos critérios de inclusão e exclusão, com amostra final de 10 (dez) pacientes submetidas ao tratamento de braquiterapia ginecológica.

A coleta de dados iniciou-se de agosto a setembro de 2021, presencialmente na Unidade. As pesquisadoras do estudo levantaram os dados das pacientes por meio de prontuários liberados, sendo analisados 60 prontuários, posteriormente 
identificado as pacientes legíveis de acordo com os critérios de inclusão e exclusão, contatou-se por meio verbal na própria Unidade nos dias de atendimento (terça, quarta e sexta feira), após esse primeiro contato, elencou-se a importância da pesquisa, esclarecendo dúvidas e dando acesso ao TCLE, exclui-se 50 pacientes por não aceitarem participar da pesquisa, assim, totalizado somente 10 participantes, sendo feita a pesquisa por meio de um questionário estruturado com as seguintes perguntas: a característica da dor antes do início da braquiterapia (período anterior a todas as sessões de braquiterapia), durante os procedimentos de preparo para a braquiterapia (entre o posicionamento da mulher na mesa ginecológica e antes do início da aplicação da radiação), durante a aplicação da braquiterapia e o procedimento de retirada dos instrumentais e depois da braquiterapia.

Os dados foram organizados mensurados por meio das condições sociodemográficas sobre as mulheres com câncer ginecológico submetidas à essa terapêutica e descrevendo as características de dor apresentados pelas pacientes antes, durante e depois do tratamento.

A análise de dados foi por meio de estatística descritiva simples, onde as amostras são visualizadas em tabelas, quadros ou gráficos, posteriormente descritos em planilha do Microsoft Excel 2013 (Lakatos \& Marconi, 2010).

Os dados foram baseados em artigos científicos nas bases de dados Scientific Electronic Library Online (SCIELO) e Literatura Latino-Americana e do Caribe em Ciências da Saúde (LILACS), sendo restringindo pelas palavras chaves definidas pelos Descritores em Saúde (DECS) Braquiterapia, Dor e Enfermagem, do ano de 2016 a 2021, exclui-se Trabalhos de Conclusão do Curso, Dissertações, Teses, casos clínicos e relatos de experiência.

\section{Resultados}

As pacientes participantes da pesquisa possuem idade diversificadas sendo $20 \%$ das participantes tem de 30 a 40 anos, $40 \%$ de 41 a 50 anos, $10 \%$ de 51 a 60 anos e 30\% mais de 60 anos. Na maioria das vezes, o desenvolvimento do câncer do colo do útero demora vários anos, no entanto, em casos raros, ele pode se desenvolver em apenas 1 ano. Essa é a razão pela qual a detecção e o diagnóstico precoce são importantes. É preciso destacar que desse quantitativo 6 (seis) pacientes apresentam tratamento de braquiterapia ginecológica completa com sedação e 4 (quatro) apresentam braquiterapia ginecológica de cúpula sem sedação.

Em relação à profissão das participantes, existe certa variedade, por exemplo, são lavradoras, aposentadas, autônomas, auxiliares de laboratórios e do lar. O grau de instrução das participantes, $40 \%$ tem fundamental incompleto, 10\% ensino médio incompleto, $20 \%$ ensino médio completo, $10 \%$ ensino superior incompleto, $10 \%$ ensino superior completo e $10 \%$ outros. No que diz respeito ao estado conjugal, $20 \%$ das participantes são solteiras, $40 \%$ casadas, $20 \%$ divorciada e $20 \%$ viúva.

Sobre a caracteristica dor, houve uma diversificação na resposta antes do início da braquiterapia (período anterior a todas as sessões de braquiterapia) conforme o Figura 1:

Figura 1 - Característica da dor antes do início da braquiterapia, Imperatriz, Maranhão, 2021

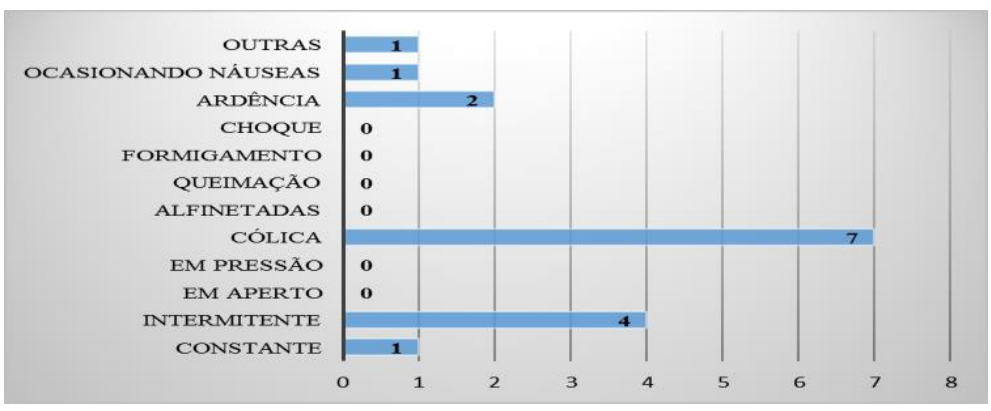

Fonte: Autores (2021). 
Durante os procedimentos de preparo para a braquiterapia (entre o posicionamento da mulher na mesa ginecológica e antes do início da aplicação da radiação) revelou-se outros achados como ardência, cólica e outras, sem dor constante ou intermitente seguindo o Figura 2:

Figura 2 - Característica da dor na realização do procedimento braquiterapia, Imperatriz, Maranhão, 2021.

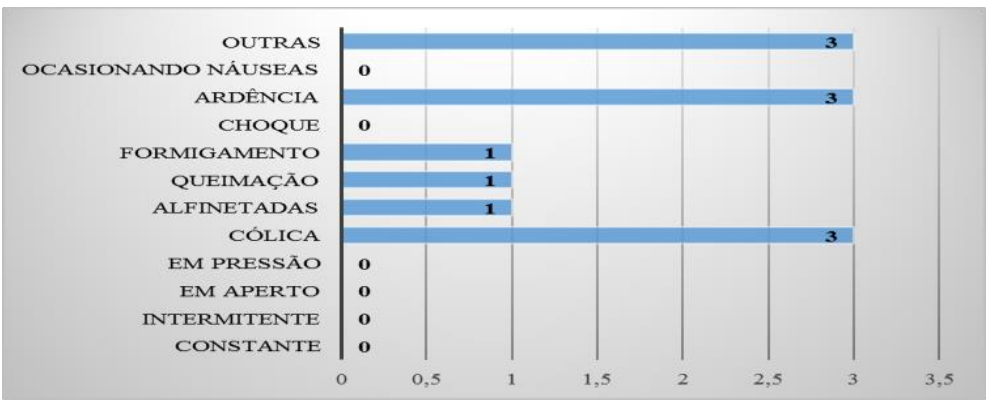

Fonte: Autores (2021).

Durante a aplicação da braquiterapia, adicinou-se queimação e tipode pressão e aperto na caracterstica da dor de acordo com Figura 3:

Figura 3 - Característica da dor durante a aplicação da braquiterapia, Imperatriz, Maranhão, 2021

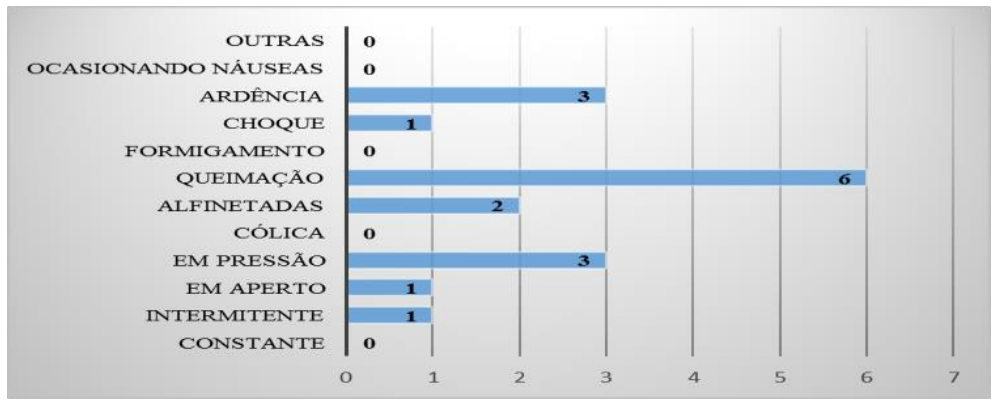

Fonte: Autores (2021).

Durante o procedimento, observou-se sintoma de queimação na maioria das pacientes submetidas a pesquisa durante retirado dos intrumentos, segundo Figura 4:

Figura 4 - Característica da dor durante o procedimento de retirada dos instrumentais, Imperatriz, Maranhão, 2021.

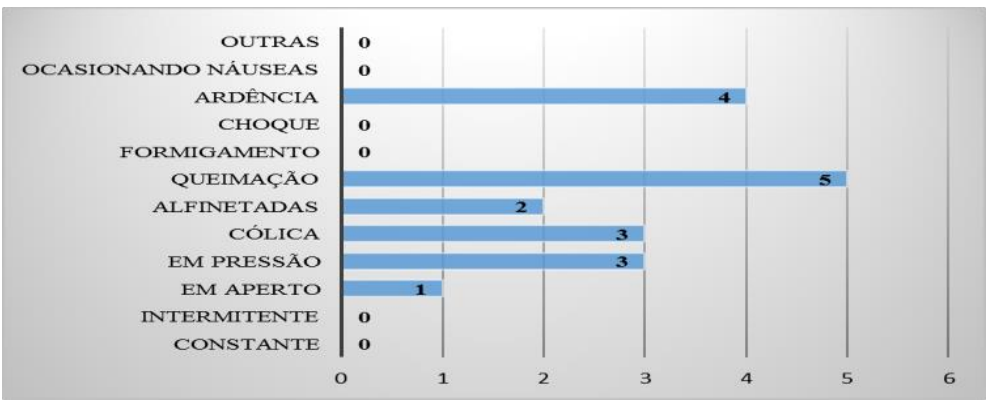

Fonte: Autores (2021).

A ardência permaneceu como principal achado juntamente com cólica, em conformidade com Figura 5: 
Figura 5 - Característica da dor durante o procedimento de retirada dos instrumentais, Imperatriz, Maranhão, 2021.

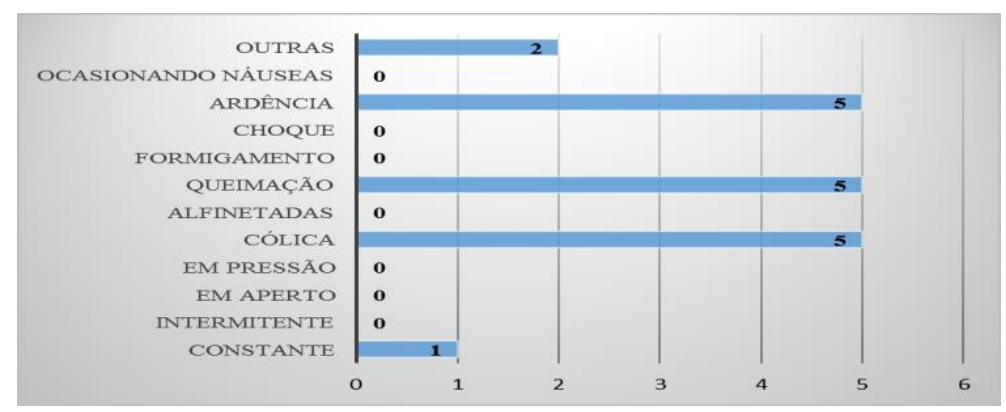

Fonte: Autores (2021).

Assim, percebeu-se que as características da dor são similares independentes da etapa do procedimento, onde cabe o enfermeiro estar atento os esses atributos.

\section{Discussão}

O câncer de colo de útero pode ser definido como alterações celulares que ocorrem de forma progressiva e consequentemente gradativa, podendo ser sintomático ou assintomático, esse é o que mais se encontra nesse tipo de câncer, atuando de forma a se desenvolver lentamente, trata-se de uma doença curável, principalmente se for descoberta de forma precoce (Campos \& Lima, 2016) (Rodrigues, Lima \& Timbó, 2017).

No que tange os fatores de risco para o câncer de colo de útero, são dos mais variados possíveis, entre eles, estão à idade, estado civil, infecções sexualmente transmissíveis, principalmente o HPV, nível socioeconômico, dentre outros (Ramalho et al., 2020). Dessa forma, torna-se cada vez mais evidente que a grande maioria deles está relacionada aos cuidados com a saúde e aos estilos de vida, muitas vezes atrapalhado pela necessidade das mulheres em trabalhar e principalmente ainda em cuidar da família.

O Câncer Cervical é uma patologia neoplásica maligna que se manifesta no aparelho reprodutor feminino, e caracteriza-se pelo surgimento de células que se multiplicam desordenadamente perdendo assim cada vez mais sua diferenciação. Sendo esse um resultado de um processo de divisão celular errada que originam o tumor, ou seja, as células cancerosas que se dividem mais rapidamente do que as normais e geralmente são bem desorganizadas. Com o tempo, podem se empilhar umas sobre as outras, formando uma massa de tecido chamada tumor. Esta neoplasia acomete a parte inferior do útero, ou seja, parte que fica no fundo da vagina, a porção enriquecida por células epiteliais (Rosa et al., 2016).

Observou-se que a percepção dolorosa não foi voluvável, sendo constante, percebendo que os efeitos relatados como dor, queimação e cólicas acontecem por ser preocedimento invasivo e estão intrisecas ao tratamento, no que tange isso, a consulta de enfermagem, que se encontra efetivada por Lei e privativa do enfermeiro, é um momento extremamente importante para as ações em saúde no contexto do cuidado oncológico, pois resulta no contato direto com o cliente, onde o cuidado individualizado se torna essencial, oportunidade também para intervenções que podem e normalmente aparecem durante o processo de adoecimento, como para as dimensões do mundo da vida da mulher com câncer (Long, Friedrich \& Jobert, 2016).

A braquiterapia é compreendida como uma radioterapia onde a radiação é entregue próxima ao tumor, através de aplicadores específicos introduzidos no canal vaginal. Mesmo sempre mostrando seus benefícios significativos, esse tratamento produz efeitos adversos que podem se tornar irreversíveis caso não sejam realizados de forma adequada. As principais reações decorrentes da braquiterapia são ardor, dor, náuseas, vômitos, infecção urinária, fraqueza, constipação, diarreia, sangramento vaginal, cansaço, hipersensibilidade vulvar e estenose vaginal (Araujo et al., 2017).

Pelo fato de ser o profissional de saúde aquele que está mais proximo da paciente, o enfermeiro acaba por 
desenvolver, além de seu papel assistencial, a atribuição do educador diante da pacinte, família e comunidade. Por meio do uso da comunicação efetiva, torna-se possível compreender as fragilidades e os medos apresentados pelas pacientes no que concerne à doença e ao tratamento, auxiliar na adaptação a essa nova condição e sanar dúvidas, objetivando um cuidado mais eficiente e adequada (Silveira, 2016).

A braquiterapia é um tratamento radioterápico consistido na utilização da radiação aplicada proximo do tumor para ser irradiado, podendo envolver cirurgia quimioterapia e hormonioterapia. O elemento utilizado para terapeutica de irídio-192, césio 137 e iodo 125. Para tratamento do câncer de colo de útero, de acordo com o estadiamento da doença, utiliza-se a braquiterapia isolada ou a radioterapia externa associada à braquiterapia (Bedell SL, Goldstein LS, Goldstein AR \& Goldstein, 2020).

Dentre os efeitos adversos ocasionados pela braquiterapia, um dos principais é a estenose vaginal, ou seja, o encurtamento e estreitamento do canal vaginal. Ela traz efeitos à mucosa vaginal, aos tecidos conectivos e aos pequenos vasos sanguíneos, resultando no desnudamento do epitélio e na diminuição do aporte sanguíneo e, consequentemente, hipóxia e desenvolvimento de teleangectasia (Pontes \& Reis, 2021).

Outros impactos sentidos ainda são na imagem corporal e autoestima, já que a doença muitas vezes afeta a aparência de forma rápida e incontrolável, o que pode acarretar em dificuldade em lidar com um "novo eu" e com os olhares e com o medo do julgamento de outras pessoas, na forma como elas estão sendo olhadas. A depressão costuma ser recorrente, além do medo da reincidência do câncer após o diagnóstico da cura (Soares et al., 2016).

Ainda a esse respeito, as pessoas com câncer ou com familiares e amigos afetados por ele, podem vivenciar diferentes maneiras de lidar com o mesmo. Muitas pessoas sentem que precisam ser fortes a fim de proteger seus amigos e familiares, outras buscam apoio e recorrem a familiares e entes queridos ou a outros sobreviventes do câncer, enquanto alguns procuram ajuda de profissionais de saúde ou mesmo recorrem a sua fé como forma de ajudá-los a sobreviver à doença (Pessi et al., 2015).

Sobre o significado do câncer na percepção dos pacientes em alguns estudos, foram registrados depoimentos que demonstram que o período do diagnóstico confirmado de câncer pode ser muito traumático. Algumas mulheres relataram que, para elas, aos olhos da sociedade, o câncer é uma doença ameaçadora à vida, que o diagnóstico e o período de tratamento foram carregados de isolamento por consequência da vergonha e do medo do julgamento e da piedade da sociedade com relação ao diagnóstico (Martelletti el al., 2021).

\section{Conclusões}

A braquiterapia tem sido uma alternativa de tratamento como forma de radioterapia onde a radiação é entregue próxima ao tumor, por meio de aplicadores específicos introduzidos no canal vaginal. Mesmo com seus benefícios significativos, esse tratamento também apresenta efeitos adversos que podem chegar a ser irreversível caso não manejado de forma adequada.

As principais reações decorrentes da braquiterapia são ardor, dor, náuseas, vômitos, infecção urinária, fraqueza, constipação, diarreia, sangramento vaginal, cansaço, hipersensibilidade vulvar e estenose vaginal, sendo muitos dessas reações pontuadas pelas participantes.

Por fim, conclui-se que é necessária uma ampliação da investigação do perfil sociodemográfico e clínico por meio de estudo quantitativo, somente assim será possível estabelecer um panorama mais amplo sobre as mulheres atendidas. Além disto, recomenda-se e que todas as informações sobre o tratamento sejam esclarecidas antes de dar início a qualquer procedimento e, ao longo das consultas de enfermagem, as informações iniciais devam ser reforçadas e ampliadas de acordo com a necessidade e interesse da mulher em braquiterapia. 
A pesquisa limitou-se aos números de participantes e também a recusa de certas pacientes a pesquisa, então a mostra denotou-se pequena para caracterização da dor de forma mais detalhada e ampla.

Esse estudo contribui para enfermagem visando subsidiar o tratamento de braquiterapia ginecológica, principalmente por ser desconhecido ao meio acadêmico, necessitando de mais aprofudamento nesse assunto, assim a importancia está pautada em conhecer a realidade dessas mulheres para promover novos conhecimentos e melhorias neste tratamento.

É necessário que outros estudos abordem a braquiterapia, visando suas consequências e efeitos ao longo prazo, além de analisar as contribuições de enfermagem nesse procedimento, visto que encontrou-se poucos artigos recente visando essa tema,

\section{Referências}

Araujo, C. R. G., et al. (2017). O fenômeno vivido por mulheres na consulta de enfermagem na braquiterapia ginecológica. Texto Contexto Enferm [online]. 26(2), 1-10.

Araujo, C. R. G et al. (2018) O Significado da dor para mulheres em braquiterapia ginecológica: abordagem fenomenológica na consulta de enfermagem. Rev Pesqui [online]. 10(3), 612-8.

Bedell, S. L., Goldstein, L. S., Goldstein, A. R., Goldstein, A. T. (2020) Cervical Cancer Screening: past, present, and future. Sex Med Ver [online]. 8(1):28-37

Brasil (2013). Resolução $\mathrm{n}^{\circ}$ 466, de 12 de dezembro de 2012. Dispõe sobre diretrizes e normas regulamentadoras de pesquisas envolvendo seres humanos. Diário Oficial [da] República Federativa do Brasil, Brasília, DF.

Campos, T. P. R., Lima C. F., Cuperschmid E. M. (2016). Balloon-based adjuvant radiotherapy in breast cancer: comparison between. Radiol Bras [online]. 49(2), 92-97.

Martelletti, L. B. S. J., Vasconcelos, S. C. C. M., Bontempo, P. S. M., et al. (2021) Avaliação da adesão ao autocuidado em mulheres submetidas à braquiterapia ginecológica. Rev Enfer Cent Oest-Min [online]. 10:e3883.

Rosa LM et al. (2016) Evaluation and classification of vaginal stenosis after brachytherapy. Texto \& Contexto Enferm [online]. 2016 [2021 cited 05 sep.] 25(2), 1-8.

Rosa LM et al. (2021) Epidemiological profile of women with gynecological cancer in brachytherapy: a cross-sectional study. Rev bras enferm [online]. 74, (5), 1-7.

Pessi, M. R., Kassulke Feuerchutte, K., Martins da Rosa, L., de Almeida Hammerschmidt, K. S., Radünz, V., \& Alvarez, A. M. (2016). Prevenção da estenose vaginal pós-braquiterapia: intervenção de enfermagem. Journal of Nursing UFPE., 10(9, 3495-3502.

Pontes, P. A., \& dos Reis, P. E. D. (2020). Validação de um manual de orientações para pacientes submetidas à braquiterapia ginecológica. Cogitare Enfermagem, 25.

Quintão, V. C. et al. (2019) Reporting guidelines: tools to increase the completeness and transparency of your anesthesiology research paper. Rev bras anestesiol [online]. 69(5), 429-431.

Lakatos, E. M., Marconi, M. A. (2010). Fundamentos de Metodologia Científica. Atlas.

Long, D., Friedrich-Nel, H. S., Joubert, G. (2016). Brachytherapy for cervical cancer: guidelines to facilitate patient-centred care in a multidisciplinary environment. S Afr J Gynaecol Oncol [online]. 8(2), 27-33

Ramalho, N. M. et al. (2020) Qualidade de vida após o câncer ginecológico: menopausa e função sexual. Rev Bras Sex Hum [online], $30(1)$, 46-53.

Silva, R. H. et al. (2021). Práticas de autocuidado e os efeitos colaterais imediatos em mulheres com câncer ginecológico em braquiterapia. Rev. Enferm. UFSM - REUFSM [online]. 2021, 11 (35), 1-22.

Rodrigues, C. F. S., Lima, F. J. C. \& Timbó, F. B. (2017). Importance of using basic statistics adequately in clinical research. Rev bras anestesiol [online]. $67(6), 619-625$.

Silva, R. M. V. D. (2015). Simulador para estudo avançado da distribuição da dose em bexiga durante procedimentos de braquiterapia ginecológica. 25 , e67109.

Silveira, C. F. et al. (2016). Qualidade de vida e toxicidade por radiação em pacientes com câncer ginecológico e mama. Esc. Anna Nery Rev. Enferm. 20(4),19.

Soares, M. L. C. A., Trezza, M. C. S. F., Oliveira, S. M. B. D., Melo, G. C. D., Lima, K. R. D. S., \& Leite, J. L. Custo da cura: vivências de conforto e desconforto de mulheres submetidas à braquiterapia. Esc. Anna Nery. 20, 317-323.

Martelletti, L. B. S. J., Vasconcelos, S. C. C. M., Bontempo P. S. M., et al. (2021). Avaliação da adesão ao autocuidado em mulheres submetidas à braquiterapia ginecológica. Rev Enfer Cent Oest-Min [online]. 10:e3883. 